\title{
Reinitiation of compensatory lung growth after subsequent lung resection
}

Lucas G. Fernandez, MD, DSc, Christopher K. Mehta, Irving L. Kron, MD, and Victor E. Laubach, PhD

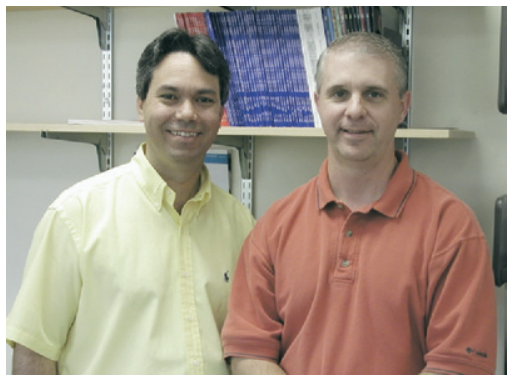

Drs Fernandez (left) and Laubach (right)
From the Department of Surgery, University of Virginia, Charlottesville, Va.

Supported by Virginia Thoracic Society Career Investigator Award CI-52-N (VEL) and National Institutes of Health grant RO1 HL67780 (VEL).

Received for publication March 9, 2007; revisions received July 6, 2007; accepted for publication July 10, 2007.

Address for reprints: Victor E. Laubach, $\mathrm{PhD}$, University of Virginia Health System, Department of Surgery, PO Box 801359, Charlottesville, VA 22908. (Email: laubach@virginia.edu)

J Thorac Cardiovasc Surg 2007;134:1300-5 $0022-5223 / \$ 32.00$

Copyright $\odot 2007$ by The American Association for Thoracic Surgery

doi:10.1016/j.jtcvs.2007.07.005
Objective: In experimental animals, pneumonectomy results in rapid, hyperplastic compensatory growth of the remaining lung. The limits of this induced growth are unknown. We tested the hypothesis that compensatory growth can be reinitiated in the same lung after subsequent lung resection.

Methods: A left thoracotomy (Sham group) or left pneumonectomy (PNX group) was performed in Sprague-Dawley rats. A third group underwent left pneumonectomy followed 4 weeks later by a bilobectomy of the right upper and middle lobes (PNX+LBX group). Four weeks after bilobectomy in the PNX+LBX group (8 weeks in the Sham and PNX groups), right ventricular pressures were measured by using the open chest technique, and total lung weight and lower plus cardiac lobe weight indices were measured. Lungs were inflation fixed at $25 \mathrm{~cm} \mathrm{H}_{2} \mathrm{O}$ to measure lobe volume index and to perform morphometric measurements on lung sections. Right ventricle/left ventricle plus septum weight index was measured as another index of pulmonary hypertension.

Results: Total lung weight index was similar in all groups. Pneumonectomy resulted in increased lower plus cardiac lobe weight and volume indices, which were significantly augmented in the PNX+LBX group. The PNX+LBX group underwent a significant increase in total volume of respiratory region, airspace, and tissue and a decrease in alveolar surface density versus the PNX group. The PNX + LBX group also had significantly increased right ventricular systolic pressure and right ventricle/left ventricle plus septum index.

Conclusion: These results demonstrate that compensatory growth can be reinitiated in lungs that had previously undergone postpneumonectomy compensatory growth. This subsequent growth, however, is more hypertrophic, and pulmonary hypertension develops despite subsequent compensatory growth.

$\mathrm{P}$ neumonectomy (PNX) or lobectomy (LBX) is often the last resort for treatment in patients who otherwise cannot be cured. These are the treatments of choice for, among other pathologies, lung cancer and congenital lobar emphysema. In the management of lung pathologies, such as cancer, the chance of local recurrences after an initial lung resection have an incidence of $1 \%$ to $5 \%$ per year, ${ }^{1,2}$ and repeat resection comes with a significant risk of death and complications compared with the initial operation. After LBX in human adults, there is a permanent loss of lung volume and function that might explain the increased risk of a repeat operation.

In experimental animals the remaining lung undergoes rapid compensatory lung growth (CLG) after PNX, which restores lung weight, volume, DNA, and protein to control levels within 3 weeks. ${ }^{3-8}$ This CLG occurs to maintain the lung's vital function of providing $\mathrm{O}_{2} / \mathrm{CO}_{2}$ exchange. In children CLG has been documented in several clinical studies ${ }^{9-11}$; however, CLG has not been documented in adult patients. Our laboratory has demonstrated in rats that CLG occurs in the left lung 


$$
\begin{aligned}
& \text { Abbreviations and Acronyms } \\
& \begin{aligned}
\text { CLG } & =\text { compensatory lung growth } \\
\mathrm{LBX} & =\text { lobectomy } \\
\mathrm{PNX} & =\text { pneumonectomy } \\
\mathrm{RVSP} & =\text { right ventricular systolic pressure } \\
\mathrm{S}_{\mathrm{v}} & =\text { alveolar surface density } \\
T V_{r a} & =\text { total volume of the respiratory airspace } \\
T V_{r t} & =\text { total volume of respiratory tissue } \\
T V_{v r} & =\text { total volume of the respiratory region }
\end{aligned}
\end{aligned}
$$

after right LBX, which is accompanied by increased vascular growth that is proportional to the amount of lung tissue removed. ${ }^{12}$ In this study, however, the removal of more than $50 \%$ of total lung mass (right upper, middle, and lower lobes) also resulted in vascular changes that lead to pulmonary hypertension.

To our knowledge, there have been no animal model studies into sequential lung resection to explore the potential of the lung to undergo more than 1 episode of compensatory growth. Our ultimate goal is to be able to manipulate those mechanisms that regulate and induce CLG (alveolarization) to benefit patients with end-stage lung disease or injury or to induce healthy lung maturation in severely premature infants. In the present study, our objectives are to (1) use a model of sequential lung resection in the adult rat, (2) describe the CLG process in this model, and (3) explore the cardiopulmonary effects of sequential lung resection.

\section{Materials and Methods \\ Animals}

Adult male Sprague-Dawley rats weighing 300 to $350 \mathrm{~g}$ were used for all experiments (Charles River, Wilmington, Mass). Facilities for animal care are accredited by the American Association for Accreditation of Laboratory Animal Care. All procedures and protocols were approved by the Institutional Animal Care and Use Committee at the University of Virginia Health System, Charlottesville, Virginia.

\section{Surgical Procedure}

The operative technique for left PNX was performed as previously described. ${ }^{12,13}$ Surgical intervention was carried out under halothane anesthesia administered through an endotracheal tube connected to a small-animal ventilator (Kent Scientific, Torlington, Conn). Animals in the PNX group $(n=9)$ underwent a left posterolateral thoracotomy, and the left lung was freed from the inferior pulmonary ligament. The lung was then delivered into the surgical wound, the hilum was tied with a medium titanium clip (HORIZON; Teleflex Medical, Research Triangle Park, NC), and the lung was excised. The chest was closed with 3-0 nylon sutures, and the skin was closed with surgical staples. Animals in the Sham group $(n=8)$ underwent a similar procedure, except the hilum was not tied and the lung was not removed. Animals in the PNX + LBX group $(n=6)$ underwent a left PNX procedure followed by 4 weeks of recovery, after which a right bilobectomy operation was performed followed by another 4 weeks of recovery. For the right bilobectomy operation, the animals underwent a right posterolateral thoracotomy, and the upper and middle lobes were ligated with a ligating titanium clip and excised. The chest was closed with 3-0 nylon sutures, and the skin was closed with surgical staples. Animals were allowed to recover from anesthesia and received postoperative analgesia in the form of buprenorphine hydrochloride $(0.03 \mathrm{mg} / \mathrm{kg}$ administered subcutaneously). All animals were allowed to feed ad libitum and were maintained in a controlled environment of normal temperature, humidity, and light cycle.

\section{Lung Harvest and Measurement of Weight and Volume Indices}

Four weeks after bilobectomy in the PNX+LBX group (8 weeks after PNX or sham operations), all groups of rats were anesthetized with a combination of ketamine and xylazine injected intraperitoneally, weighed, and intubated through a tracheotomy, and exposure of the organs was obtained by using a bilateral anterior sternothoracotomy. The animals were rapidly exsanguinated by means of renal vein division, and the right lung (or left and right lungs in the Sham group) was removed, blotted dry, and weighed. The upper and middle lobes were then ligated and carefully dissected out, and the lower plus cardiac lobe weight was obtained. Lower and cardiac lobes received intratracheal instillation of $4 \%$ paraformaldehyde to a pressure of $25 \mathrm{~cm} \mathrm{H}_{2} \mathrm{O}$ to obtain lower plus cardiac lobe volumes. The trachea was then tied under pressure, and the total lung volume was obtained by means of volume displacement, as described by Scherle. ${ }^{14}$ Lung volumes (in milliliters) and weights (in grams) were expressed as a ratio to the final body weights of the animals (in grams) to obtain the volume and weight indices.

\section{Lung Morphometry}

Lung morphometry was performed by using a 3-level sampling technique ${ }^{15,16}$ and point-counting technique, ${ }^{17}$ as previously described by our laboratory. ${ }^{18,19}$ A 42-point test reticule (lattice with 85- $\mu \mathrm{m}$ grid lines) attached to a Nikon Eclipse E400 microscope was used. This technique involved 3 levels of study performed on hematoxylin and eosin-stained slides (5 random fields per animal). The first level of analysis, which is usually performed under gross inspection, was not performed because of the small size of the rat lung and the lack of accurate differentiation at the gross level.

The second level of analysis was performed at $40 \times$ magnification. The number of lattice points that fell on intra-acinar air space and their intervening tissue was counted and designated as $\mathrm{P}_{\mathrm{r}}$. Nonrespiratory regions, which included extra-acinar airways and vessels larger than $0.5 \mathrm{~mm}$ in diameter, were ignored. The volume density of the respiratory region $\left(V_{v r}\right)$ was calculated by using the equation $V_{v r}=P_{r} / 42 \times 100$, which represents the percentage of lung comprised of respiratory (alveolar) tissue and airspace.

The third level of analysis was performed under $200 \times$ magnification. The number of lattice points that overlap the respiratory airspaces was designated as $P_{r a}$. The volume density of the respiratory airspace $\left(V_{r a}\right)$ was then calculated by using the following equation: $V_{r a}=P_{r a} / 42 \times 100$. In addition, the number of lattice 
points that fell in the respiratory tissue was counted and designated as $P_{r t}$. The volume density of respiratory tissue $\left(V_{r t}\right)$ was then calculated by using the following equation: $V_{r t}=P_{r t} / 42 \times 100$. These values represent the percentage of the lung that is comprised of respiratory alveolar airspace $\left(V_{r a}\right)$ and tissue $\left(V_{r t}\right)$, respectively.

Alveolar surface density $\left(S_{v}\right)$, which expresses the alveolar surface area per unit volume, was determined by using the equation $S_{v}=2 / d \times\left(I_{s} / P_{p}\right)$, where $d$ is defined as the length of the lattice grid line $(85 \mu \mathrm{m}), I_{s}$ is the number of lattice test lines that intercepted the airspace-epithelial interfaces, and $P_{p}$ is defined as the total number of lattice test points on the lung parenchyma (42). The total volume of the respiratory region $\left(T V_{v r}\right)$ was calculated as $T V_{v r}=V_{v r} \times V_{l}$, where $V_{l}$ is defined as the total lung volume. $T V_{v r}$ is a measure of the total volume of alveoli and intervening gas exchange tissue. The total volume of the respiratory airspace $\left(T V_{r a}\right)$ was calculated as $T V_{r a}=V_{v r} \times V_{r a} \times V_{l}$. This is a measure of alveolar airspace volume in the lung. Total volume of respiratory tissue $\left(T V_{r t}\right)$ was calculated as $T V_{r t}=V_{v r} \times V_{r t} \times V_{l}$. This is a measure of alveolar tissue volume in the lung.

Right Ventricular Hypertrophy and Systolic Pressure Right ventricular systolic pressures (RVSPs) were measured immediately before death, as previously described. ${ }^{20}$ Briefly, the rats were anesthetized with ketamine and xylazine and placed in a supine position. The transducer was calibrated before study, and RVSP was measured by means of direct intracardiac puncture with a 21-gauge needle. At death, hearts were removed and dissected to isolate the free wall of the right ventricle from the left ventricle and septum. The ratio of right ventricular weight to left ventricular plus septal weight was used as an index of right ventricular hypertrophy, which develops as a result of pulmonary hypertension.

\section{Statistical Analysis}

Data are presented as the mean \pm standard error of the mean. Statistical analysis was performed with the SPSS 14.0 for Windows software program (Apache Software Foundation, Forest Hill, $\mathrm{Md})$. Comparisons were made with 1-way analysis of variance and the post-hoc Bonferroni test.

\section{Results}

\section{Total Lung Weight and Lower Plus Cardiac Lobe Weight Indices}

The total lung weights and the lower plus cardiac lobe weights were measured in the PNX $+\mathrm{LBX}$ group at 4 weeks after bilobectomy ( 8 weeks after PNX or sham operations). Total lung weight index (left plus right lungs in the Sham group, right lung in the PNX group, and lower plus cardiac lobes in the PNX+LBS group) was similar in all groups (Figure 1,A), which is indicative of compensatory growth after PNX or PNX+LBX to equal the left plus right lungs in the Sham group. When only lower plus cardiac lobe weight indices were compared, the PNX group had a significantly higher lower plus cardiac lobe weight index compared with the Sham group $(1.91 \pm 0.06$ vs $1.40 \pm 0.07$, $P<.05)$. In addition, the lower plus cardiac lobe weight index for the PNX+LBX group $(3.63 \pm 0.28)$ was signif-
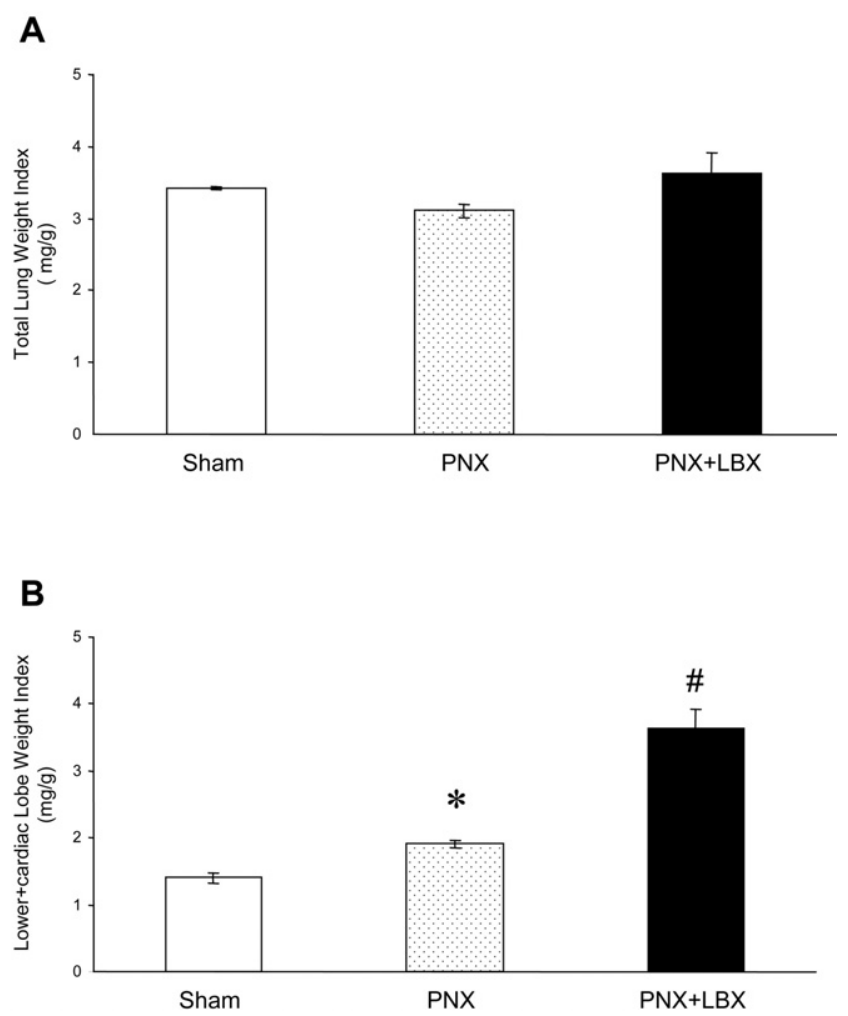

Figure 1. A, Total lung weight index (left plus right lungs in the Sham group, right lung in the pneumonectomy [PNX] group, and lower plus cardiac lobes in the pneumonectomy plus lobectomy [PNX+LBS] group) was similar in all groups. B, Lower plus cardiac lobe weight index was significantly increased in the PNX group versus that in the Sham group $\left({ }^{*} P<.0001\right)$. In addition, lower plus cardiac lobe weight index of the PNX+LBX group was significantly higher than that of the PNX group (\#P $<.0001)$.

icantly increased above that in the PNX group $(P<.0001$; Figure 1, $B$ ).

\section{Lower Plus Cardiac Lobe Volume Index}

The lower plus cardiac lobe volume indices were measured 4 weeks after bilobectomy in the PNX+LBX group (8 weeks after PNX or sham operations). The PNX group showed a significant increase in lower plus cardiac lobe volume index versus that seen in the Sham group (22.52 \pm 0.77 vs $16.40 \pm 0.44, P<.0001$, Figure 2 ). The lower plus cardiac lobe volume index in the PNX+LBX group (31.70 $\pm 0.71)$ was further significantly increased versus that seen in the PNX group $(P<.0001$, Figure 2$)$.

\section{Right Ventricular Hypertrophy and Systolic Pressure} There was no change in right ventricular hypertrophy or RVSP after PNX alone (Figure 3). Subsequent resection of the upper and middle lobes (PNX+LBX group) resulted in 


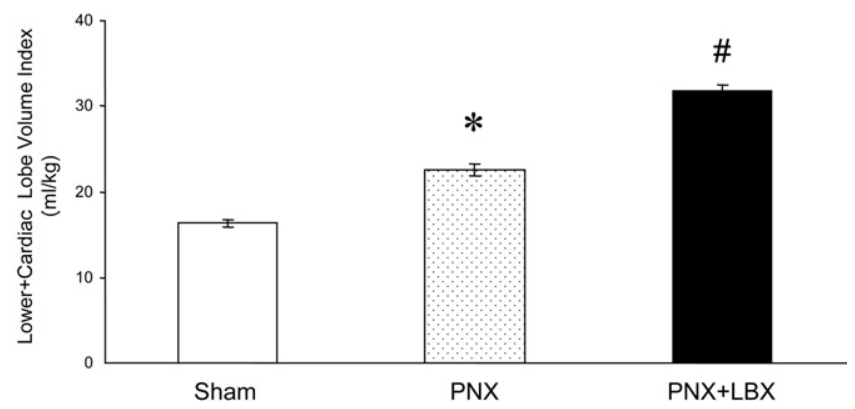

Figure 2. Changes in lower plus cardiac lobe volume index. Lower plus cardiac lobe volume index was significantly increased in the pneumonectomy (PNX) group versus the Sham group $\left({ }^{*} \boldsymbol{P}<.05\right)$. In addition, lower plus cardiac lobe volume index for the pneumonectomy plus lobectomy $(P N X+L B X)$ group was significantly higher than that in the PNX group (\#P<.0001).

significant pulmonary hypertension, as shown by a significant increase of $\operatorname{RVSP}(P=.01$; Figure $3, A)$, as well as a significant increase in right ventricular hypertrophy (Figure $3, B)$.

\section{Lung Histology and Morphometry}

Gross histologic evaluation of lung sections showed that lung architecture appeared normal in all the groups (Figure 4), with no signs of edema, cellular infiltration, or thickening of alveolar septa. Detailed morphometric analysis was used to measure the $S_{v}, T V_{v r}, T V_{r a}$, and $T V_{r t}$ values in the lower lobes of each group (these values were also similar for the cardiac lobes, data not shown). These results are shown in Table 1 . At 8 weeks, $S_{v}$ was significantly less in the PNX group $(P<.001)$ and was even further reduced significantly in the PNX+LBX group $(P<.05)$. The PNX group had significantly higher $T V_{v r}$ and $T V_{r a}$ values when compared with the Sham group $(P<.001)$, and both $T V_{v r}$ and $T V_{r a}$ values were significantly augmented in the PNX+LBX group when compared with the PNX group $(P$ $<$.0001). The PNX group had higher $T V_{r t}$ values than the Sham group, which did not reach statistical significance; however, values in the PNX+LBX group were significantly higher than those in the Sham group $(P<.05)$.

\section{Discussion}

The treatment of lung disease at times requires PNX, LBX, or both, resulting in compensatory growth of the remaining lung in infants and young children. ${ }^{9-11}$ In experimental animals compensatory growth of a mature lung occurs after PNX, resulting in restoration of lung volume, mass, and function in the remaining lung. In addition, restoration of whole lung levels of protein, RNA, DNA, collagen, and elastin, along with essentially normal lung cell populations, also occurs. ${ }^{3,21,22}$ Although there is no dispute that the
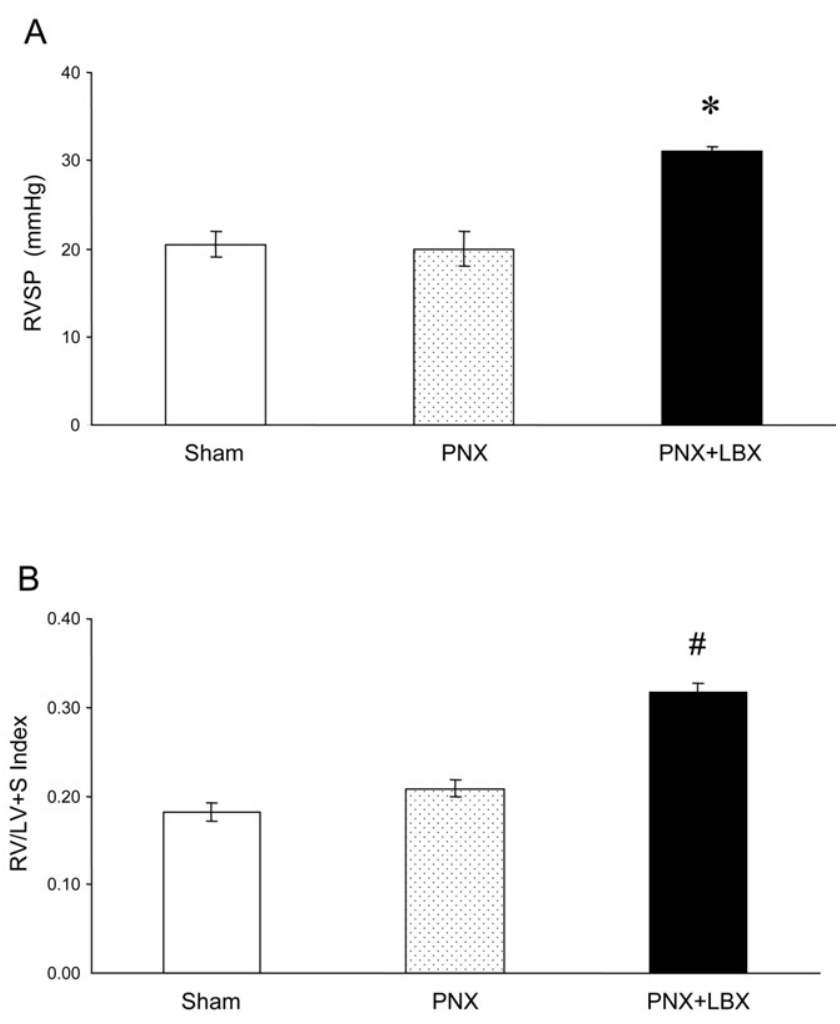

Figure 3. Pulmonary hypertension is induced after pneumonectomy plus lobectomy (PNX+LBX) operations. A, Right ventricular systolic pressure (RVSP) was significantly increased in the PNX+LBX group versus the pneumonectomy (PNX) and Sham groups $\left({ }^{*} P=.01\right)$. $B$, Ratios of right ventricle to left ventricle plus septum weights $(R V / L V+S)$, an assessment of right ventricular hypertrophy, were compared between groups. RV/LV+S was significantly increased in the PNX+LBX group compared with that seen in the PNX and Sham groups $(\# P<.0001)$.

remaining tissue undergoes compensatory growth after PNX, the nature and extent of this growth are not fully understood. Understanding the controlling factors and dynamics of CLG could permit the minimization of the stresses experienced by the remaining lung and lead to methods to shorten the time required for new tissue growth.

In this study we evaluated the growth response in lungs 4 weeks after PNX + LBX and compared these with lungs 8 weeks after PNX or sham operations. The period of 4 weeks after each operation was chosen to be a time in which lung growth and healing will have occurred in completion because it has been shown that post-PNX lung growth reaches a plateau between 10 and 21 days. In the present study total lung weight remained unchanged between the groups after the operations (Figure 1,A), and when lower plus cardiac lobe weights and volumes were examined, it was evident that compensatory growth occurred in both the PNX and 


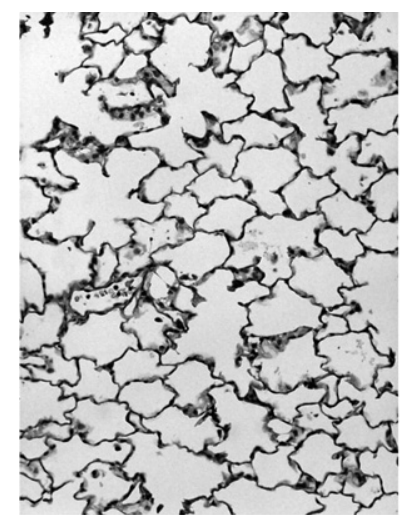

Sham

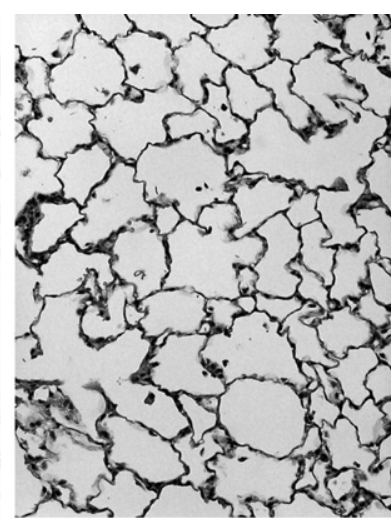

PNX

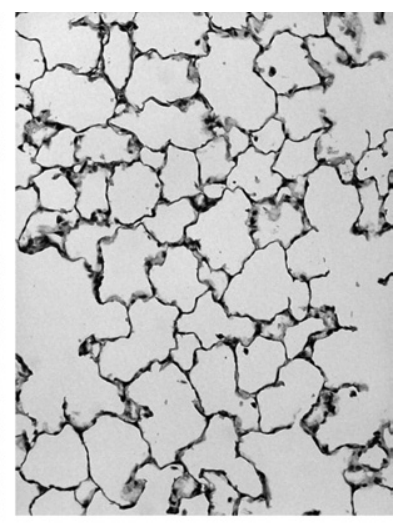

PNX+LBX
PNX + LBX groups. Most interesting, a significant augmentation in the lower plus cardiac lobe weight and volume indices was observed in the PNX+LBX group when compared with the PNX group (Figures 1, B, and 2), indicating that CLG was reinitiated after the second bilobectomy operation.

CLG was also evident after morphometric analysis, which revealed that PNX increases $\mathrm{TV}_{\mathrm{vr}}, \mathrm{TV}_{\mathrm{ra}}$, and $\mathrm{TV}_{\mathrm{rt}}$ values after PNX (Table 1), confirming previous results. ${ }^{18,19}$ Interestingly, a further significant augmentation in these indices was observed in the PNX + LBX group compared with that seen in the PNX group. The $S_{v}$ value, however, was significantly reduced after PNX and further reduced after PNX + LBX. A decrease in $\mathrm{S}_{\mathrm{v}}$ value is likely a result of larger alveoli, which can be explained by alveolar hypertrophy in these groups. This alveolar hypertrophy is not obvious on the basis of lung histology (Figure 4), which appears normal in all groups and requires morphometric analysis to detect. A significant decrease in $S_{v}$ value was not observed in previous studies after PNX, ${ }^{18,23}$ which was probably due to the 3 -week post-PNX period in these studies as opposed to the 8-week post-PNX period in the current study. These extra 5 weeks likely entailed further normal lung growth and expansion, leading to decreased $\mathrm{S}_{\mathrm{v}}$ values. We have previously shown that PNX enhances lung weight and volume by means of alveolar hyperplasia, ${ }^{13,18,23}$ and we can postulate that both alveolar hyperplasia and hypertrophy occur in the PNX $+\mathrm{LBX}$ group, resulting in increased $\mathrm{TV}_{\mathrm{rt}}$ and decreased $S_{v}$ values. This finding of increased $T_{v r}$ and $\mathrm{TV}_{\mathrm{ra}}$ values combined with increased $\mathrm{TV}_{\mathrm{rt}}$ values and lower plus cardiac lobe volumes indicates that CLG after subsequent lung resection appears to be very dynamic, entailing both alveolar hypertrophy and hyperplasia, perhaps with hypertrophy being a more significant component than after PNX alone.

In the present study there was a significant increase in RVSP and right ventricular hypertrophy after PNX plus LBX when compared with that seen in the Sham and PNX groups, indicating that pulmonary hypertension developed. This finding is consistent with our previous study in which the removal of 3 right lobes (approximately 53\% of total lung mass) resulted in CLG of the left lung but accompanied by pulmonary hypertension. ${ }^{12}$ The left lung represents approximately $37 \%$ of the total lung mass in the rat, and the right upper and middle lobes account for approximately $24 \%$. Our findings suggest that the bilobectomy performed 4 weeks after left PNX (which represents approximately $61 \%$ of the original total lung mass resected) was significant enough to cause pulmonary hypertension, despite the compensatory growth after each operation.

In summary, bilobectomy after PNX reinitiated a CLG response in the remaining lung of adult rats. In contrast to PNX alone, this growth involved a greater hypertrophic response, and pulmonary hypertension developed despite

Table 1. Morphometric analysis of the Sham, PNX, and PNX+LBX groups

\begin{tabular}{llccc}
\hline & $\mathbf{S}_{\mathbf{v}}\left(\mathbf{c m}^{2} / \mathbf{c m}^{3}\right)$ & $\mathbf{T V}_{\mathbf{v r}}(\mathbf{m L})$ & $\mathbf{T V}_{\mathbf{r a}}(\mathbf{m L})$ & $\mathbf{T V}_{\mathbf{r t}}(\mathbf{m L})$ \\
\hline Sham group & $173.20 \pm 9.97$ & $7.36 \pm 0.36$ & $5.27 \pm 0.32$ & $1.79 \pm 0.12$ \\
PNX group & $139.08 \pm 6.97 \dagger$ & $10.69 \pm 0.20 \dagger$ & $8.16 \pm 0.35 \dagger$ & $2.30 \pm 0.23$ \\
PNX+LBX group & $119.13 \pm 7.72^{*}$ & $15.13 \pm 0.84 \ddagger$ & $12.25 \pm 0.72 \ddagger$ & $2.54 \pm 0.20^{*}$
\end{tabular}

Values are presented as means \pm standard error of the mean. $P N X$, Pneumonectomy; $P N X+L B X$, pneumonectomy plus lobectomy; $S_{v \prime}$ alveolar surface density; $T V_{v r}$ total volume of respiratory region; $T_{\mathrm{ra}}$, total volume of respiratory airspace; $T V_{\mathrm{rt}}$, total volume of respiratory tissue. ${ }^{*} P<.05$ versus the $S$ ham group; $\uparrow P<.001$ versus the Sham group; $¥ P<.0001$ versus the PNX and Sham groups. 
subsequent CLG. It is likely that pulmonary hypertension resulted from the amount of lung parenchyma removed in the PNX+LBX group rather than the compensatory growth. To our knowledge, this is the first time that a model of sequential lung resection has been used to study the modulation of post-PNX CLG. Our study provides novel information regarding the potential for compensatory growth after a second lung resection. A better understanding of the various modulators of lung growth has enormous clinical application because scenarios such as reduced-size lung transplantation and pulmonary insufficiency can benefit from the modulation and induction of lung growth.

\section{References}

1. Pairolero PC, Williams DE, Bergstralh EJ, Piehler JM, Bernatz PE, Payne WS. Postsurgical stage I bronchogenic carcinoma: morbid implications of recurrent disease. Ann Thorac Surg. 1984;38:331-8.

2. Deschamps C, Pairolero PC, Trastek VF, Payne WS. Multiple primary lung cancers. Results of surgical treatment. J Thorac Cardiovasc Surg. 1990;99:769-77.

3. Kuboi S, Mizuuchi A, Mizuuchi T, Taguchi T, Thurlbeck WM, Kida K. DNA synthesis and related enzymes altered in compensatory lung growth in rats. Scand J Clin Lab Invest. 1992;52:707-15.

4. Buhain WJ, Brody JS. Compensatory growth of the lung following pneumonectomy. J Appl Physiol. 1973;35:898-902.

5. Brody JS, Burki R, Kaplan N. Deoxyribonucleic acid synthesis in lung cells during compensatory lung growth after pneumonectomy. Am Rev Respir Dis. 1978;117:307-16.

6. Brody JS. Time course of and stimuli to compensatory growth of the lung after pneumonectomy. J Clin Invest. 1975;56:897-904.

7. Berger LC, Burri PH. Timing of the Quantitative Recovery in the Regenerating Rat Lung. Am Rev Respir Dis. 1985;132:777-83.

8. Hsia CC, Herazo LF, Fryder-Doffey F, Weibel ER. Compensatory lung growth occurs in adult dogs after right pneumonectomy. J Clin Invest. 1994;94:405-12.

9. Frenckner B, Freyschuss U. Pulmonary function after lobectomy for congenital lobar emphysema and congenital cystic adenomatoid malformation. A follow-up study. Scand J Thorac Cardiovasc Surg. 1982;16:293-8.
10. Nakajima C, Kijimoto C, Yokoyama Y, Miyakawa T, Tsuchiya Y, Kuroda $\mathrm{T}$, et al. Longitudinal follow-up of pulmonary function after lobectomy in childhood-factors affecting lung growth. Pediatr Surg Int. 1998;13:341-5.

11. McBride JT, Wohl ME, Strieder DJ, Jackson AC, Morton JR, Zwerdling RG, et al. Lung growth and airway function after lobectomy in infancy for congenital lobar emphysema. J Clin Invest. 1980;66:96270.

12. Le Cras TD, Fernandez LG, Pastura PA, Laubach VE. Vascular growth and remodeling in compensatory lung growth following right lobectomy. J Appl Physiol. 2005;98:1140-8.

13. Fernandez LG, Le Cras TD, Ruiz M, Glover DK, Kron IL, Laubach VE. Differential vascular growth in postpneumonectomy compensatory lung growth. J Thorac Cardiovasc Surg. 2007;133:309-16.

14. Scherle W. A simple method for volumetry of organs in quantitative steorology. Mikroskopie. 1970;26:57-60.

15. Davies P. Morphologic and morphometric techniques for the detection of drug- and toxin-induced changes in lung. Pharmacol Ther. 1991; 50:321-36.

16. Wandel G, Berger LC, Burri PH. Morphometric analysis of adult rat lung after bilobectomy. Am Rev Respir Dis. 1983;128:968-72.

17. Gil J. Models of lung disease. New York: Marcel Dekker; 1990.

18. Kaza AK, Kron IL, Leuwerke SM, Tribble CG, Laubach VE. Keratinocyte growth factor enhances post-pneumonectomy lung growth by alveolar proliferation. Circulation. 2002;106(suppl):I120-4.

19. Leuwerke SM, Kaza AK, Tribble CG, Kron IL, Laubach VE. Inhibition of compensatory lung growth in endothelial nitric oxide synthasedeficient mice. Am J Physiol Lung Cell Mol Physiol. 2002;282: L1272-8.

20. Fagan KA, Fouty BW, Tyler RC, Morris KG Jr, Hepler LK, Sato K, et al. The pulmonary circulation of homozygous or heterozygous eNOS-null mice is hyperresponsive to mild hypoxia. $J$ Clin Invest. 1999;103:291-9.

21. McAnulty RJ, Staple LH, Guerreiro D, Laurent GJ. Extensive changes in collagen synthesis and degradation during compensatory lung growth. Am J Physiol. 1988;255:C754-9.

22. Koh DW, Roby JD, Starcher B, Senior RM, Pierce RA. Postpneumonectomy lung growth: a model of reinitiation of tropoelastin and type I collagen production in a normal pattern in adult rat lung. Am J Respir Cell Mol Biol. 1996;15:611-23.

23. Kaza AK, Kron IL, Kern JA, Long SM, Fiser SM, Nguyen RP, et al. Retinoic acid enhances lung growth after pneumonectomy. Ann Thorac Surg. 2001;71:1645-50. 